\title{
复杂表面小尺度凝结现象真实感仿真
}

\author{
施佳俊, 李晨, 王长波 ${ }^{*}$ \\ (华东师范大学计算机科学与技术学院 上海 200062)
}

(cbwang@cs.ecnu.edu.cn)

\begin{abstract}
摘 要: 凝结是自然界中很常见的一种现象, 其真实感仿真对虚拟现实、影视特效及游戏娱乐等领域都有重要的意 义. 针对传统的基于物理的仿真方法难以保持凝结小尺度液滴细节的问题，提出一种基于光滑粒子流体动力学与自 适应流体隐式粒子法耦合的仿真方法. 首先基于光滑粒子流体动力学离散建模空气的热传导, 并辅以相对湿度模型 和露点描述相变过程; 然后结合基于八叉树的自适应背景网格和流体隐式粒子法仿真凝结液滴, 突出了小尺度液滴 高精度的细节; 最后引人表面张力、黏附力与阻力, 逼真地仿真了复杂固体表面上液滴的运动. 实验结果表明, 该方 法可以真实、高效地反映复杂固体表面的凝结现象.
\end{abstract}

关键词：流体仿真; 自适应网格; 凝结现象; 小尺度液滴仿真

中图法分类号: TP391.41 DOI: 10.3724/SP.J.1089.2021.18543

\section{Small-Scale Condensation Simulation on Complicated Surface}

\author{
Shi Jiajun, Li Chen, and Wang Changbo* \\ (School of Computer Science and Technology, East China Normal University, Shanghai 200062)
}

\begin{abstract}
Vapor condensation is a typical phenomenon in the nature, whose realistic simulation is of great significance to virtual reality, video effects, game entertainment, etc. Traditional physically-based methods failed to preserve the small-scale details during condensation. A novel hybrid framework combining smoothed particle hydrodynamics and adaptive fluid implicit particle method is proposed. The heat transfer of air is modeled with smoothed particle hydrodynamics, with phase transition controlled by relative humidity and dew point. The fluid implicit particle method is combined with an octree-based adaptive grid to achieve high-resolution details of small-scale drops. Finally, surface tension, adhesion and resistance are introduced to simulate the motion of drops on complicated surfaces realistically. It is demonstrated that proposed method provides plausible results of condensation on intricate surfaces efficiently through several experiments.
\end{abstract}

Key words: fluid simulation; adaptive grid; condensation; small-scale drop simulation

凝结是自然界中很常见的一种现象, 其真实 感仿真在虚拟现实、影视特效及游戏娱乐等多个方 向都有广泛的应用. 潮湿的空气遇冷, 或温度降低 到临界温度之下时, 就会出现水滴附着在固体表 面, 如玻璃窗、树叶和杯壁上, 形成凝结液滴. 因 此, 仿真凝结现象需要考虑热传导、相变以及凝结
液滴运动等过程, 涉及气体、液体和固体三者之间 的交互. 由于凝结液滴尺度小且受力复杂, 如何真 实、高细节地仿真凝结现象仍是一大挑战性课题.

目前在图形学领域, 主流的基于物理的流体 仿真方法分为拉格朗日法、欧拉法和混合方法 3 种. 其中, 拉格朗日法主要通过粒子离散流体场,

收稿日期: 2020-08-05; 修回日期: 2020-09-17. 基金项目：国家自然科学基金(62072183, 61672237,62002121). 施佳俊(1995一), 男, 硕士研究生, 主要研究方向为流体仿真; 李晨(1990-), 男, 博士, 博士后, 主要研究方向为流体仿真; 王长波(1976一), 男, 博 士，教授，博士生导师，CCF 会员，论文通讯作者，主要研究方向为计算机图形学、数字媒体、虚拟现实. 
追踪每个粒子上的物理量仿真流体, 如光滑粒子流 体动力学 (smoothed particle hydrodynamics, SPH) ${ }^{[1]}$. SPH 易于实现和理解，且能够捕捉泡沫等表面细 节，但流场的不可压缩性不易保持，也难以通过粒 子重构出光滑的表面. 欧拉法 ${ }^{[2]}$ 以交错网格离散 Navior-Stokes 方程，通过观察空间中固定点上流 体的物理量随时间的变化描述流体场. 该方法能 够保证稳定、不可压缩的仿真效果，然而具有计算 量大、内存消耗高等缺点, 且往往因数值耗散和立 方体网格结构损失大量细节，最终导致边界走样. 以流体隐式粒子法(fluid implicit particle method, FLIP)方法 ${ }^{[3]}$ 为代表的混合方法在欧拉法的基础上 引人粒子, 保证了低数值耗散, 但在高精度仿真时 仍有网格方法固有的内存消耗和计算量大的问题.

凝结现象仿真的主要难点在于, 如何精确地 计算出凝结的位置并对小尺度的凝结液滴保留较 高细节. 使用网格法难以精确地计算液滴凝结的 位置; 而为了表现凝结液滴在复杂固体表面上真 实感的运动, 则需要使用高精度的网格法准确地 计算液滴的形状，这又导致了巨大计算量和高内 存消耗的问题. 另外, 表面张力、黏附力和阻力对 固体表面小尺度的液滴仿真有重要的作用，使用 传统的网格法由于边界走样, 很快就会导致液滴 失真.

针对上述问题，本文采用 SPH 方法对空气建 模，以计算精确的凝结位置，并用露点温度协同相 对湿度模型标记相变的发生；对于凝结液滴，则以 FLIP 方法结合自适应的背景网格，在保证复杂固 体表面液滴细节的同时，有效地降低了计算量和 内存消耗. 此外, 本文还考虑了凝结液滴所受的表 面张力、黏附力和阻力, 加强了液滴在固体表面运 动的细节。

\section{1 相关工作}

在计算机图形学领域，对于流体仿真已有相 当多的成果. 由于 SPH 等基于粒子的方法计算精 度往往受制于粒子数量，而基于网格的欧拉法则 有网格精度和数值耗散带来的流场失真和变形 ${ }^{[4]}$, 近年来学者逐渐倾向于使用混合方法仿真流体, 以结合二者的优势, FLIP 方法即是其中一种. FLIP 方法在通过欧拉网格计算保证流场不可压缩性的 同时引人粒子, 降低了数值耗散, 并加强了表面的 小尺度细节. FLIP 方法最初由 Zhu 等 ${ }^{[3]}$ 引人流体仿 真中用来仿真沙子的运动，之后被广泛地应用于
仿真大规模的液体飞溅 ${ }^{[5]}$ 和二相流 ${ }^{[6]}$ 等流体现象. 同时, 针对 FLIP 方法的一些缺点, 许多学者也提 出改进, 如 Ferstl 等 ${ }^{[7]}$ 通过只在表面窄带范围内采 样粒子，有效地降低了粒子插值引起的计算消耗; Ando 等 ${ }^{[8]}$ 动态细分了液体表面的 FLIP 粒子, 以保 持对流体边缘薄膜的真实感仿真.

凝结是一种常见的相变过程. 物质由于热传 导导致其温度变化, 温度达到一定的阈值, 物质就 会发生形态转变, 如冰块融化、熔岩凝固和水汽凝 结等都是常见的相变现象. Stomakhin 等 ${ }^{[9]}$ 基于物 质点方法引入潜热构建模型, 仿真了物质融化的 过程; 张申帆等 ${ }^{[10]}$ 基于 $\mathrm{SPH}$ 方法考虑动态黏度, 逼真地仿真了熔岩、蜡烛等凝固和融化的现象; Ding 等 ${ }^{[11]}$ 也以物质点方法对巧克力和黄油等融化 的现象进行了探究. 相对地, 凝结现象则较少有人 建模仿真. 张泰滺等 ${ }^{[12]}$ 提出相对湿度模型和露点 建模水汽的凝结, 突出了水汽凝结过程的物理性; Hochstetter 等 ${ }^{[13]}$ 结合欧拉法、SPH 方法和表面纹理 仿真了液滴蒸发和凝结的过程, 得到了较真实的 效果.

在仿真诸如表面液滴此类小尺度的现象时， 往往由表面张力、黏附力和阻力等宏观场景下几乎 可忽略的力起决定性作用, 其中表面张力通常通 过最小化表面的曲率实现. Clavet 等 ${ }^{[14]}$ 在 SPH 框架 中引人表面张力和基于距离的黏附力, 得到了能 吸附于固体表面的黏性液滴; Akinci 等 ${ }^{[15]}$ 进一步探 究了表面张力、内聚力和黏附力三者协同对液滴形 状和运动的作用; 徐士彪等 ${ }^{[16]}$ 结合菲涅尔模型, 仿真了液滴受基于平均曲率的表面张力影响下的 效果. 而在欧拉方法中, 表面张力往往通过对液滴

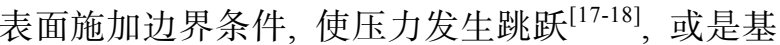
于体积保持平均曲率流方法 ${ }^{[19]}$ 进行计算.

基于网格的仿真方法的一个重大缺陷是由于 计算精度上升导致的计算量和内存消耗的急剧增 长, 因而通过均匀网格仿真高精度的流体现象往 往是不可行的. 对此, 现有的工作往往采用自适应 的方法降低仿真的资源消耗. Losasso 等 ${ }^{[20]}$ 首先将 八叉树结构引入流体仿真中, 通过网格细化技术 在捕捉表面细节的同时，大大降低了计算量和内 存消耗; Setaluri 等 ${ }^{[21]}$ 和 Aanjaneya 等 ${ }^{[22]}$ 在此基础上 进一步提出 SPGrid 结构, 利用稀疏网格和分页机 制在存储和缓存调度层面减轻了内存和计算负担; Ferstl 等 ${ }^{[23]}$ 结合八叉树结构和六面体的有限元方 法, 取得了不错的仿真效果; Goldade 等 ${ }^{[24]}$ 将自适 应方法引人黏性流体的仿真中, 缓解了黏度计算 
时由于大量速度自由度导致的庞大线性矩阵的计 算量; Lyu 等 ${ }^{[25]}$ 在 FLIP 仿真二相流的框架中加人 网格自适应方法, 在保证仿真质量的基础上提升 了仿真的速度. 受此启发, 本文为了在有限的运算 资源下得到凝结液滴高精度的表面细节，在以 FLIP 方法高保真地仿真液滴的同时，借助自适应 网格兼顾存储资源的消耗.

\section{2 自适应的 FLIP 方法}

基于网格的流体仿真的算法复杂度与网格精 度息息相关，增加 1 倍网格精度往往导致需要 8 倍 的内存空间和 8 倍以上的计算时间，故直接以均匀 网格进行高精度的小尺度液滴仿真是不可行的. 受 Lyu 等 ${ }^{[25]}$ 工作的启发，本文为使仿真达到预期 的精度，引人自适应的背景网格代替均匀网格，同 时借助 FLIP 粒子保证准确的流体表面.

\subsection{FLIP 方法}

FLIP 方法是一种将欧拉法和拉格朗日法混合 的方法，它利用粒子保持流场的几何结构，用背景 网格施加外力和边界条件并保持流场的不可压缩 性. 其物理控制方程为经典的 Navior-Stokes(NS)方 程, 即

$$
\left\{\begin{array}{l}
\frac{\partial \boldsymbol{u}}{\partial t}=-(\boldsymbol{u} \cdot \nabla) \boldsymbol{u}-\frac{1}{\rho} \nabla p+\boldsymbol{f} \\
\nabla \cdot \boldsymbol{u}=0
\end{array} .\right.
$$

其中， $\boldsymbol{u}$ 为速度； $t$ 为时间； $\rho$ 为密度； $p$ 为压力； $\boldsymbol{f}$ 为外力. 而 $\nabla \cdot \boldsymbol{u}=0$ 约束了流场的不可压缩性, 表示流体的速度场散度为 0 .

在迭代计算流体的运动时，先以粒子水平集 方法 ${ }^{[26]}$ 确定流场的区域和边界, 再以背景网格施 加外力并投影得到无散的流场，最后插值得到每 个粒子的速度并更新位置. 为了使流场具有一定 的黏性, FLIP 方法常与质点网格法(particle-in-cell, $P I C)^{[27]}$ 进行一定比例的混合, 即

$$
\mathbf{u}_{i}=(1-\alpha) \mathbf{u}_{i}^{\mathrm{PIC}}+\alpha \mathbf{u}_{i}^{\mathrm{FLIP}} \text {. }
$$

其中, $\boldsymbol{u}_{i}$ 为粒子 $i$ 的速度; $\boldsymbol{u}_{i}^{\mathrm{PIC}}$ 为粒子位置在背景 网格上插值得到的速度; $\boldsymbol{u}_{i}^{\mathrm{FLIP}}$ 为粒子当前速度与 粒子位置在背景网格上插值得到的速度之和与施加 外力前速度的差值; $\alpha \in[0,1]$ 为二者间的混合比例.

\section{2 基于八叉树的自适应网格}

依据 Losasso 等 ${ }^{[20]}$ 的思想，本文基于八叉树设 计了背景网格的层次结构. 在以交错网格作欧拉 计算的同时，通过在八叉树节点的 8 个顶点上的存
储速度、水平集等物理量简化八叉树空间中的插值 运算. 当需要相互转换时, 面中心的物理量可直接 由面的 4 个顶点上相应的值平均得到, 而顶点的物 理量则可根据 Setaluri 等 ${ }^{[21]}$ 的方法计算.

为了突出小尺度液滴的细节，应使液滴的表 面在整个运算中一直以最细的网格表示，而其余 部分可使用较粗网格简化计算. 类似 Ferstl 等 ${ }^{[23]}$ 的工作，本文根据水平集的值在表面附近维护了 一条细化窄带, 如图 1 所示. 在每个仿真迭代中, 背景网格都会基于 FLIP 粒子水平集不断分裂与合 并八叉树的节点, 以保证细化窄带内的网格都是 最细层次网格，且调节时间步长使表面在平流后 仍处在窄带内.

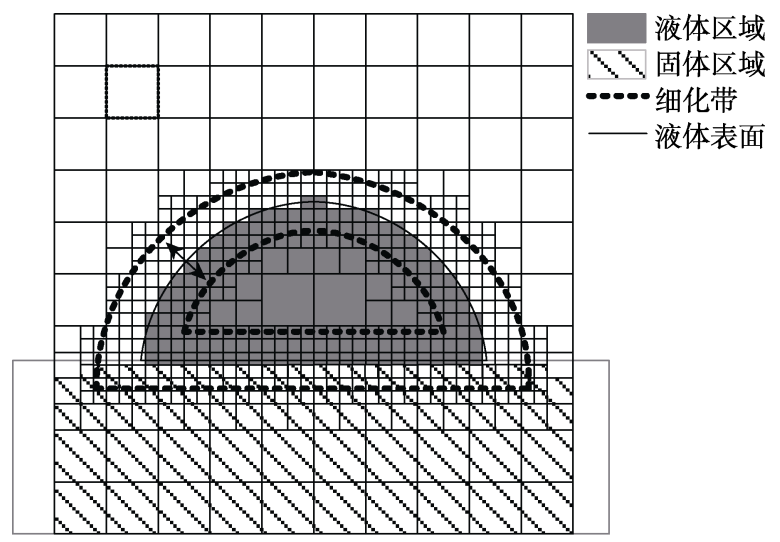

图 1 自适应网格结构示意图

在求解压力泊松方程时，由于相邻网格间尺 度可能不同, 需要重新考虑散度算子和压力梯度 的计算. 类似 Losasso 等 ${ }^{[20]}$ 的工作，对每个网格的 散度算子，有 $V_{\text {cell }} \nabla \cdot \boldsymbol{u}=\sum_{\text {faces }}\left(\boldsymbol{u}_{\text {face }} \cdot \boldsymbol{n}\right) S_{\text {face }}$. 其中， $V_{\text {cell }}$ 为网格的体积; $\boldsymbol{u}_{\text {face }}$ 为面中心的速度分量; $\boldsymbol{n}$ 为面向外的法向量; $S_{\text {face }}$ 为面的面积. 而相邻网 格间的梯度算子为 $p_{x}=\left(p_{2}-p_{1}\right) / \Delta_{12}$. 其中, $p_{1}$ 和 $p_{2}$ 为相邻 2 个网格的压力; $\Delta_{12}$ 取 2 个网格中较大 网格大小 $\Delta_{\text {large }}$ 、较小网格大小 $\Delta_{\text {mall }}$ 或二者的平均 值等均不影响其收玫性, 本文取 $\Delta_{12}=1.54_{\mathrm{small}}$. 最 后, 由大型对称线性方程组通过共轭梯度法求解 得到压力。

\subsection{FLIP 粒子与背景网格的耦合}

在 FLIP 方法中，粒子与网格的耦合主要在于 计算水平集以及速度插值. 在自适应改变局部网 格分辨率时, 需要水平集来标记细化窄带的大小 和位置. 本文以粒子水平集方法 ${ }^{[26]}$ 计算流体水平 集, 并测试每个节点的顶点是否处于细化窄带内. 
若有顶点处于窄带内，则对其作分裂操作，分裂后 新增的顶点物理量由原有 8 个顶点值平均得到; 若 同一父节点的 8 个子节点均无顶点在窄带内, 则对 它们进行合并操作. 在完成网格自适应之后, 本文 根据水平集标记流体区域，并将粒子的速度按距 离插值函数映射到流体区域节点的所有顶点之上.

每次迭代中，本文在完成背景网格上所有运 算后，按传统的 FLIP 方法进行平流来更新粒子的 速度和位置. 对自适应背景网格上的空间插值, 参 考 Setaluri 等 ${ }^{[21]}$ 的方法, 本文首先将网格中的点投 影到对应的左右 2 个面上, 以修正的双线性插值通 过面顶点上的物理量得到面上投影点的值, 再线 性插值 2 个投影点得到最终的结果.

\section{3 基于 SPH 的空气热传导与相变}

凝结现象真实感仿真中 2 个非常重要的因素 是温度的变化和凝结时机的确定. 虽然用欧拉法 仿真空气中的热量等属性变化的物理准确性更好, 但对高精度的凝结现象, 大量的空气网格会增加 不必要的计算量和内存消耗, 且难以准确地计算 凝结所在的位置. 因此, 本文选择 SPH 方法仿真 空气.

\subsection{SPH 方法}

传统 SPH 方法的基本思想是将连续流场离散 成粒子系统来描述流体的运动. 每个粒子都有各 种物理属性, 包括质量 $m$ 、密度 $\rho$ 、位置 $\boldsymbol{x}$ 、速度 $\boldsymbol{u}$ 和压力 $p$ 等. 在每次迭代计算中, 每个粒子 $i$ 的属 性值由其邻居粒子 $N_{i}$ 的属性以支持半径为 $h$ 的各类 光滑核函数 $W$ 插值得到, 再通过 Navior-Stokes 方程 计算加速度来更新粒子位置，插值公式为

$$
A_{i}=\sum_{j \in N_{i}} A_{j} \frac{m_{j}}{\rho_{j}} W\left(\left\|\boldsymbol{x}_{i}-\boldsymbol{x}_{j}\right\|, h\right) .
$$

其中, $A_{i}$ 和 $A_{j}$ 分别为粒子 $i$ 和 $j$ 的某一属性值.

\section{2 热传导}

根据 Cleary 等 ${ }^{[28]}$ 的工作，本文空气粒子间热传 导的公式经 SPH 离散计算得到，对于空气粒子 $i$, 有

$$
\frac{\partial T_{i}}{\partial t}=\frac{1}{\rho_{i} C_{i}} \sum_{j \in N_{i}} \frac{4 \psi_{i} \psi_{j}}{\psi_{i}+\psi_{j}} V_{j} \frac{T_{i}-T_{j}}{\left\|\boldsymbol{x}_{i}-\boldsymbol{x}_{j}\right\|} \nabla W\left(\left\|\boldsymbol{x}_{i}-\boldsymbol{x}_{j}\right\|, h\right) .
$$

其中, $T$ 为温度; $C$ 为比热容; $\psi$ 为热传导系数, $V$ 为体积.

而空气与液体、固体间的热传导由于液体和固 体在系统中以网格形式存在, 需要考虑粒子与网 格间的耦合，根据 Hochstetter 等 ${ }^{[13]}$ 的工作，空气粒
子 $i$ 的温度变化为

$$
\frac{\partial T_{i}}{\partial t}=\frac{1}{\rho_{i} C_{i}} S_{i} \sum_{g \in N_{i}} \frac{4 \psi_{i} \psi_{g}}{\psi_{i}+\psi_{g}}\left(T_{i}-T_{g}\right) \beta_{i g} .
$$

其中, $g$ 为粒子 $i$ 邻居集合 $N_{i}$ 中的一个格点； $S_{i}$ 为粒子与液体、固体的接触面积; $\beta_{i g}$ 为衡量网格 与粒子间热传导的基于距离的插值函数. 为了进 一步减少内存的消耗，本文假定固体温度为一个 定值，从而简化了固体网格温度的计算.

\section{3 相对湿度模型和露点计算}

露点指在一定气压和水汽含量的情况下使空 气冷却达到饱和的温度, 当空气温度降低到露点 以下时, 形成凝结液滴. 现有的工作往往只考虑了 空气的温度而没有重视空气中水汽含量的变化. 本文沿用张泰滺等 ${ }^{[12]}$ 的方法建立空气相对湿度模 型来仿真水汽含量变化, 对露点计算的物理性作 了更精致的建模. 以下简单复述其思想.

相对湿度是衡量一定气压下空气中水汽含量 的一种指标. 空气粒子相对湿度的变化由湿度扩 散模型给出，即

$$
\frac{\partial H_{i}}{\partial t}=k_{\text {diff }} \sum_{j \in N_{i}} m_{j} \frac{H_{j}-H_{i}}{\rho_{j}} \nabla^{2} W\left(\left\|\boldsymbol{x}_{i}-\boldsymbol{x}_{j}\right\|, h\right) .
$$

其中, $k_{\text {diff }}$ 为空气湿度扩散系数; $H$ 为相对湿度.

根据 August-Roche-Magnus 公式，一定气压下空 气的露点可由相对湿度和温度近似计算得到, 即

$$
\left\{\begin{array}{l}
T_{\mathrm{dp}}=\frac{c \gamma(T, H)}{b-\gamma(T, H)} \\
\gamma(T, H)=\ln \left(\frac{H}{100}\right)+\frac{b T}{c+T}
\end{array} .\right.
$$

其中, $T_{\mathrm{dp}}$ 为露点温度; 常量系数 $b=17.67 ; c=$ $243.5^{\circ} \mathrm{C}$

当空气粒子达到露点而发生凝结时, 会在距 凝结粒子最近的固体表面生成一个半球状液滴, 其大小由相对湿度决定, 且凝结后降低的湿度会 由湿度扩散模型得到平衡.

\section{4 小尺度凝结液滴的仿真}

发生凝结现象时，会在固体表面生成小尺度 的凝结液滴. 除了考虑重力以外, 表面张力以及固 体表面的黏附力和阻力也是影响液滴形状和运动 趋势非常重要的因素. 因这 3 个力都作用于流体表 面, 本文直接将它们作为外力作用到细化窄带内 的液滴网格. 本节将详细介绍以自适应 FLIP 方法 仿真小尺度液滴时对其受力的处理. 


\section{1 表面张力}

表面张力可以通过对流体表面施加一个大小 为 $\sigma \kappa$ 的边界条件, 使表面压力产生跳跃来得到 ${ }^{[17]}$. 其中， $\sigma$ 为表面张力系数， $\kappa$ 是由流体水平集计算 得到的表面曲率，即 $\kappa=\nabla \cdot(\nabla \phi /|\nabla \phi|)$. 其中， $\phi$ 为 水平集的值.

\section{2 黏附力和阻力}

为了表现小尺度凝结液滴附着于玻璃等固体 表面并沿表面流动的现象，一些 SPH 方法常加人 黏附力来表现这样的效果, 如文献[14-15], 但在网 格法仿真中很少有工作探究黏附力对表面液滴的 影响. 受上述工作启发, 本文将黏附力建模为流固边界一个指向固体表面的力，即

$$
\boldsymbol{f}_{\text {adhesion }}=k_{\text {adhesion }} \Delta t \frac{d\left(d-d_{\text {adhesion }}\right)}{d_{\text {adhesion }}^{2}} \boldsymbol{n} .
$$

其中, $\boldsymbol{f}_{\text {adhesion }}$ 为黏附力; $k_{\text {adhesion }}$ 为固体对水的黏 附系数; $d$ 为到固体表面的距离; $d_{\text {adhesion }}$ 为最大 黏附影响距离; $\boldsymbol{n}$ 为表面法向量.

同时，在固体表面运动的液滴也会受到一个 平行于表面的阻碍液滴运动的阻力，即

$$
\boldsymbol{u}_{\mathrm{T}}=\max \left(0, \boldsymbol{u}_{\mathrm{T}}\left[1-\frac{\mu|\boldsymbol{u} \cdot \boldsymbol{n}|}{\left|\boldsymbol{u}_{\mathrm{T}}\right|}\right]\right) .
$$

其中, $\boldsymbol{u}_{\mathrm{T}}$ 为流-固边界速度的平行分量； $\mu$ 为库仑 摩擦系数. 本文通过综合考虑黏附力和阻力, 可以 较逼真地仿真液滴于固体表面流下时的尾迹和垂 落等现象.

\section{5 算法实现}

本文空气以 SPH 框架仿真, 使用 CUDA 对其 进行优化, 并用基数排序加速邻居查找的过程. 凝 结液滴和固体表面则由自适应网格表示. 本文的 自适应网格结构由多层级指针数组构成, 最粗层 标记为 $L_{\max }$, 最细层标记为 $L_{0}$. 网格相邻 2 层分 辨率增加 1 倍，故上一层的每个节点对应下一层的 8 个子节点, 逻辑上呈八叉树结构, 且其中只有叶 节点挂载运算所需的存储空间, 从而最小化内存 消耗. 本文中每个叶节点含 18 个单精度浮点数通 道, 由速度和水平集值的存储以及共轭梯度法和 顶点运算等共用，并使用 OpenMP 对网格计算进 行了加速.

当空气粒子凝结时，会在最接近粒子的固体 表面生成一个的由 FLIP 粒子构成的半球状液滴, 其半径为 $r$. 此时如果直接在固体表面新增液滴会
显得比较突元, 因此, 本文加人了一个等待队列以 平滑过渡液滴的生成过程. 液滴生成时, 新增的 FLIP 粒子不会立即加人运算, 而是先作为一个整 体置于等待队列中, 在每次迭代中由 0 不断增大队 列中液滴的半径, 当半径达到 $r$ 时再将这些 FLIP 粒子加人运算. 渲染时, 以等待队列中所有液滴现 有的半径 $r_{\text {current }}$ 标记其可见粒子, 并与 FLIP 粒子 一同通过 Marching Cube 算法 ${ }^{[29]}$ 得到表面. 具体算 法如下.

输人．等待队列 $\boldsymbol{W}_{\text {list }}$, FLIP 粒子集合 $\boldsymbol{F}$.

输出，等待队列 $\boldsymbol{W}_{\text {list }}^{\prime}$, FLIP 粒子集合 $\boldsymbol{F}^{\prime}$.

Step1. 更新 $\boldsymbol{W}_{\text {list }}$ 中元素 $w$ 的现有半径

$$
r_{\text {current }}=r_{\text {current }}+\Delta r \text {. }
$$

Step2. 对元素 $w$ 的现有半径 $r_{\text {current }}$ 进行判断: 若 $r_{\text {current }} \geqslant r$, 则将 $w$ 移出 $\boldsymbol{W}_{\text {list }}$, 并将其粒子加人 $\boldsymbol{F}$ 中.

Step3. 重复 Step1 和 Step2 直到 $\boldsymbol{W}_{\text {list }}$ 的队列尾, 得 到新的等待队列 $\boldsymbol{W}_{\text {list }}^{\prime}$ 和 FLIP 粒子集合 $\boldsymbol{F}^{\prime}$.

在每次通过 FLIP 粒子更新水平集后，需要通 过八叉树的分裂与合并使流体表面处于细化窄带 内, 并使窄带内网格达到最高精度, 以突出液滴的 表面细节. 本文基于半径为 $h_{\text {refine }}$ 的细化窄带的自 适应分裂合并算法如下.

输人. 八叉树层级网格 $\boldsymbol{G}$, 水平集 $\phi$, 细化窄 带半径 $h_{\text {refine }}$.

输出. 八叉树层级网格 $G^{\prime \prime}$.

Step1. 对 $\boldsymbol{G}$ 从 $L_{\text {max }}$ 到 $L_{1}$ 进行分裂操作得到 $\boldsymbol{G}^{\prime}$ :

Step1.1. 对 $L$ 层中每个叶节点 $g$ 的 8 个顶点水平 集 $\phi_{q 1} \sim \phi_{g 8}$ 进行判断, 若 8 个值中有任意一个值 $\phi_{q}$ 满足 $\left|\phi_{g}\right|<h_{\text {refine }}$, 则分裂 $g$;

Step1.2. $L=L-1$.

Step2. 对 $\boldsymbol{G}^{\prime}$ 从 $L_{0}$ 到 $L_{\text {max- }-1}$ 进行合并操作得到 $\boldsymbol{G}^{\prime \prime}$ :

Step2.1. 对 $L$ 层中每 8 个相同父节点的叶节点 $g_{1} \sim g_{8}$ 的 27 个顶点水平集 $\phi_{91} \sim \phi_{g 27}$ 进行判断, 若 27 个 值 $\phi_{g}$ 均满足 $\left|\phi_{g}\right| \geqslant h_{\text {refine }}$ ，则合并 $g_{1} \sim g_{8}$;

Step2.2. $L=L+1$

其中, 分裂操作会在下一层生成 8 个叶节点, 在顶点的物理量传递给叶节点后将当前节点转为 中间节点并删除其存储空间，叶节点其余 19 个顶 点值可由原有 8 个顶点值平均得到; 合并操作则会 在将 8 个顶点值传递给父节点后, 将父节点转为叶 节点并删除 8 个子节点.

\section{6 实验结果与分析}

本文的实验平台为 Intel Core(TM) i5-4590 
CPU, 8 GB 内存, NVIDIA GTX960 GPU, CUDA8.0. 本文共做了 5 个实验，以检验本文方法的各个细节.

图 2 展示了本文通过等待队列平滑过渡单个 液滴生成的效果. 可以看到液滴随时间不断增大， 最后由于重力开始运动的整个过程. 显然, 直接在 固体表面生成一个较大的液滴是非常突兀的，而 通过本文的等待队列方法逐渐在固体表面生成夜 滴, 则更加符合自然界中凝结的过程, 使仿真更加 自然.

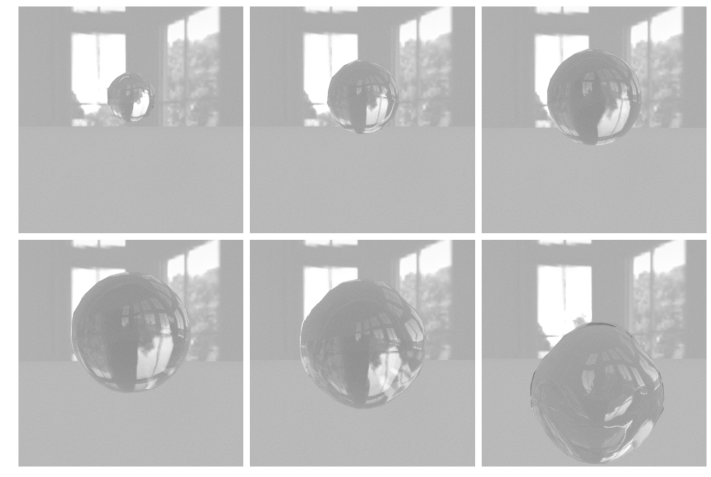

图 2 液滴逐渐生成的过程

为了验证本文液滴受力模型中不同表面材质 对于液滴运动的影响, 设计了图 3 所示的 4 个场 景。在保证初始条件相同的情况下，通过调节模 型中黏附力和阻力等参数表现液滴在玻璃、塑料、 岩石及木材等不同固体材质表面的运动趋势. 从 图 3 可以观察到, 不同的固体材质对液滴运动的 快慢、液滴的尾迹的长短和宽度都有不同程度的 影响. 该实验说明, 本文的液滴受力模型能够正 确地反映小尺度凝结液滴在不同固体材质表面运 动的细节.

本文引人黏附力是为了与其他作用力协同, 以表现液滴附着于固体表面流下和垂落的效果, 从而真实地反映自然界中的场景. 为了体现黏附 力和表面张力对固体表面液滴运动的作用，本文 对水流在表面张力和黏附力作用下沿球面缓缓流 下，并仿真了垂落和滴下的整个过程，如图 4 所示， 4 幅图分别为仿真的第 150, 200,250 和 300 帧. 图 $4 \mathrm{a}$ 中处于球体侧面的水流由于黏附力能够抵消重 力的部分分量, 而使水流能吸附于球体表面; 而 当流到球体下部时, 则因重力影响而缓缓垂落, 如图 $4 \mathrm{~b}$ 所示; 在图 $4 \mathrm{c}$ 和图 $4 \mathrm{~d}$ 中, 垂落的水流受 到表面张力的影响，前端逐渐收缩成球形，并最 终发生断裂. 可见，本文所引人的黏附力可以在 与表面张力和重力等作用力协同下，保证了仿真 的真实感.

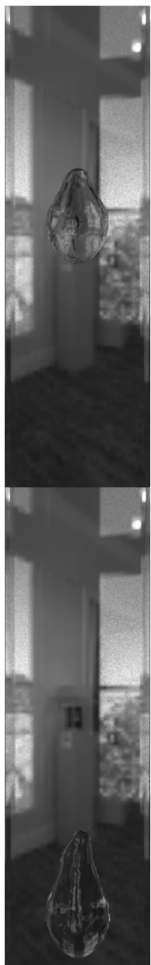

a. 玻璃

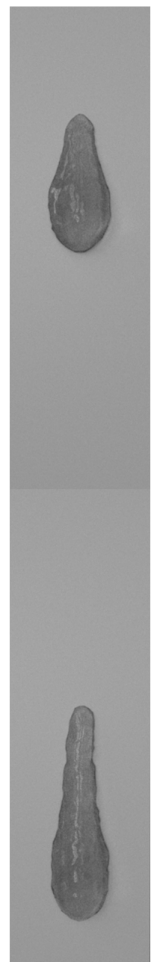

b. 塑料

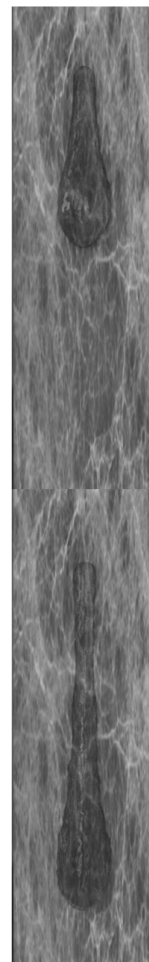

c. 岩石

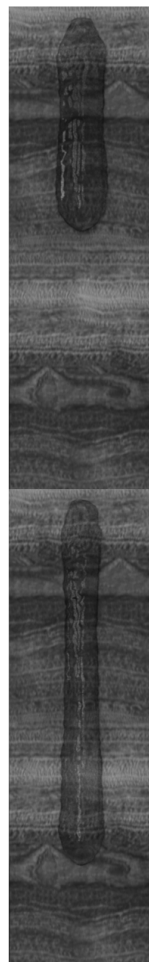

d. 木材
图 3 不同固体材质下液滴运动对比

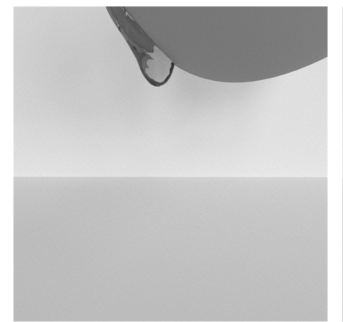

a. 第 150 帧

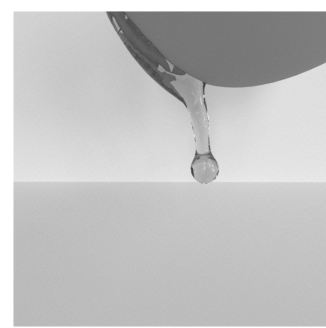

c. 第 250 帧

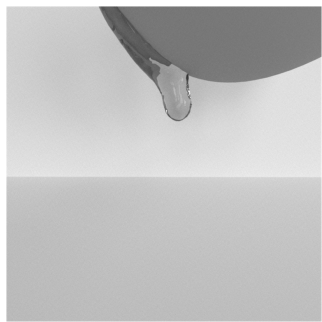

b. 第 200 帧

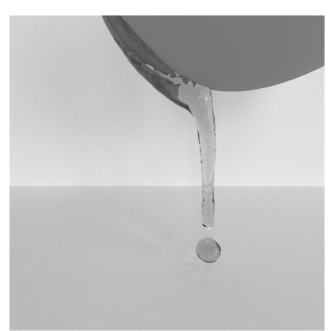

d. 第 300 帧
图 4 水流沿球面垂落的过程

图 5a 以本文框架仿真了在镜面上的凝结现象, 热空气不断与低温镜面进行热交换导致局部温度 降低到露点以下, 从而出现水汽凝结于镜面之上. 由于本文采用了等待队列的液滴生成策略, 凝结 液滴在生成后逐渐变大, 最后受重力、表面张力和 阻力等作用逐步沿镜面流下并形成细长的尾迹. 对比图 $5 \mathrm{~b}$ 中以高度场方法 ${ }^{[30]}$ 得到的液滴运动现 象，本文仿真效果在尾迹和液滴形态等方面更加 
逼真且人工痕迹较少. 同时，本文方法能够支持更 高的分辨率, 且避免图 $5 \mathrm{~b}$ 中出现的锯齿状的液滴 表面.

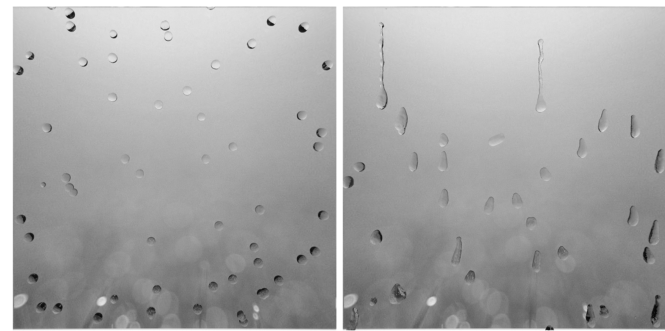

a. 本文

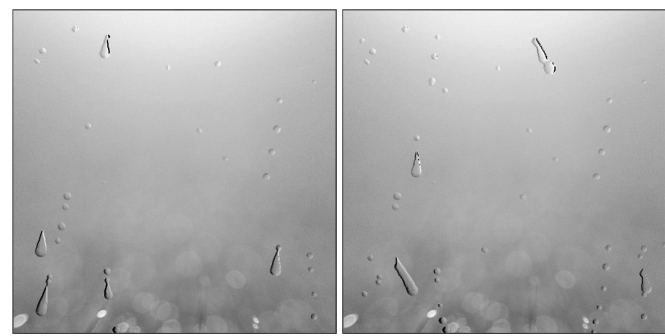

b. 高度场 ${ }^{[30]}$

图 5 不同方法仿真镜面凝结现象对比

在图 6中，本文设计了水汽凝结于玻璃杯外壁 上的实验场景，通过对比体现了本文液滴受力模 型中黏附力和阻力对于凝结现象细节保持的重要 作用. 图 6a 中, 由于缺少黏附力和阻力的作用, 凝 结液滴在表面运动时缺乏尾迹等交互细节，且液 滴无法吸附于外壁之上，导致仿真效果不理想. 而 在图 $6 \mathrm{~b}$ 中, 黏附力和阻力的加人, 大大提升了凝 结液滴与复杂固体表面的交互细节.

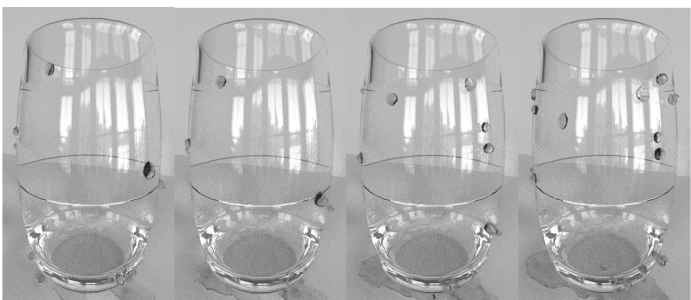

a. 未考虑黏附力和阻力

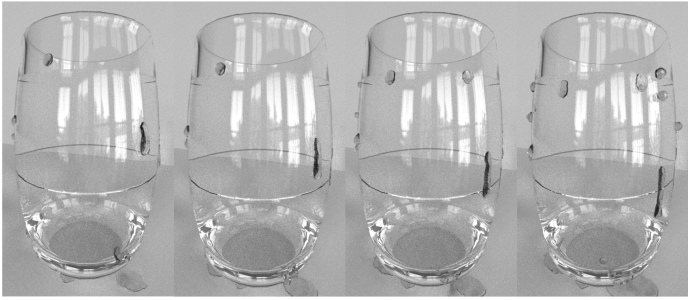

b. 考虑黏附力和阻力

图 6 玻璃杯外壁凝结的现象对比

表 1 给出了本文场景的运行效率和内存占用 情况. 可以看到，本文自适应仿真框架可以大大
降低背景网格中叶节点的个数, 从而使仿真能 在保持流体表面分辨率较高的情况下将内存消 耗控制在较低的程度; 同时, 仿真的运行速度也 较快。

表 1 本文运行效率和内存占用情况

\begin{tabular}{|c|c|c|c|c|c|}
\hline \multirow[b]{2}{*}{ 场景 } & \multirow{2}{*}{$\begin{array}{c}\text { 场景 } \\
\text { 分辨率 }\end{array}$} & \multicolumn{2}{|c|}{ 叶节点数 } & \multirow{2}{*}{$\begin{array}{c}\text { 内存 } \\
\text { 占用/MB }\end{array}$} & \multirow{2}{*}{$\begin{array}{c}\text { 运行 } \\
\text { 时间/s }\end{array}$} \\
\hline & & $\begin{array}{c}\text { 自适应 } \\
\text { 网格 }\end{array}$ & 均匀网格 & & \\
\hline 球面垂落 & $\begin{array}{c}128 \times 128 \times \\
128\end{array}$ & 435744 & 2097152 & 210.025 & 7.212 \\
\hline 镜面凝结 & $\begin{array}{c}512 \times 512 \times \\
64\end{array}$ & 3216224 & 16777216 & 1310.781 & 59.807 \\
\hline $\begin{array}{l}\text { 玻璃杯外 } \\
\text { 壁凝结 }\end{array}$ & $\begin{array}{c}256 \times 256 \times \\
256\end{array}$ & 1867736 & 16777216 & 1084.084 & 50.125 \\
\hline
\end{tabular}

7 结 语

本文基于 SPH 方法对空气进行离散建模，考 虑空气间以及空气与固、液间的热传导, 结合相对 湿度模型计算露点, 对水汽凝结的过程进行了充 分的探究. 本文在引人自适应的 FLIP 方法在突出 小尺度凝结液滴在复杂表面滑落细节的同时，大 大降低了计算代价. 此外, 本文加人黏附力和阻力 完善了表面液滴的受力模型, 提升了凝结液滴形 状与运动的真实感与准确性.

在后续工作中，本文还需进一步完善自适应 框架以减少内存消耗并加速仿真, 如尝试使用稀 疏多重网格结构压缩存储, 或加人 NB-FLIP方法 ${ }^{[7]}$ 并用多重网格共轭梯度法加速粒子与网格的插值 和压力计算. 此外, 使用 SPH 方法仿真空气虽能 准确得到凝结点, 但粒子数量不足会导致其物理 准确性较差，而粒子数量上升又会影响仿真效率, 因此，本文将继续探索如何将空气仿真加人自适 应框架，以获得更准确的仿真效果.

\section{参考文献(References):}

[1] Ihmsen M, Orthmann J, Solenthaler B, et al. SPH fluids in computer graphics[C] //Proceedings of Eurographics. Aire-la-Ville: Eurographics Association Press, 2014: 21-42

[2] Stam J. Stable fluids[C] //Proceedings of the 26th Annual Conference on Computer Graphics and Interactive Techniques. New York: ACM Press, 1999: 121-128

[3] Zhu Y N, Bridson R. Animating sand as a fluid[J]. ACM Transactions on Graphics, 2005, 24(3): 965-972

[4] Liu Youquan, Liu Xuehui, Zhu Hongbin, et al. Physically based fluid simulation in computer animation[J]. Journal of Computer-Aided Design \& Computer Graphics, 2005, 17(12): 2581-2589(in Chinese) 
(柳有权, 刘学慧, 朱红斌, 等. 基于物理的流体模拟动画综 述 [J]. 计算机辅助设计与图形学学报, 2005, 17(12): 2581-2589)

[5] Gerszewski D, Bargteil A W. Physics-based animation of large-scale splashing liquids[J]. ACM Transactions on Graphics, 2013, 32(6): Article No.185

[6] Boyd L, Bridson R. MultiFLIP for energetic two-phase fluid simulation[J]. ACM Transactions on Graphics, 2012, 31(2): Article No.16

[7] Ferstl F, Ando R, Wojtan C, et al. Narrow band FLIP for liquid simulations[J]. Computer Graphics Forum, 2016, 35(2): 225-232

[8] Ando R, Thurey N, Tsuruno R. Preserving fluid sheets with adaptively sampled anisotropic particles[J]. IEEE Transactions on Visualization and Computer Graphics, 2012, 18(8): 1202-1214

[9] Stomakhin A, Schroeder C, Jiang C, et al. Augmented MPM for phase-change and varied materials[J]. ACM Transactions on Graphics, 2014, 33(4): Article No.138

[10] Zhang Shenfan, Li Chen, Wang Changbo. Realistic fluid simulation considering heat conduction and variable viscosity [J]. Journal of Computer-Aided Design \& Computer Graphics, 2017, 29(7): 1186-1194(in Chinese) (张申帆, 李晨, 王长波. 考虑热传导与动态黏度的真实感 流体仿真 [J]. 计算机辅助设计与图形学学报, 2017, 29(7): 1186-1194)

[11] Ding M Y, Han X C, Wang S, et al. A thermomechanical material point method for baking and cooking[J]. ACM Transactions on Graphics, 2019, 38(6): Article No.192

[12] Zhang Taiyou, Shi Jiajun, Wang Changbo. Simulation of condensation phenomenon based on air heat exchange[J]. Journal of Computer-Aided Design \& Computer Graphics, 2017, 29(10): 1794-1801(in Chinese)

(张泰滺，施佳俊，王长波. 考虑空气热交换的凝结现象真 实感仿真[J]. 计算机辅助设计与图形学学报, 2017, 29(10): 1794-1801)

[13] Hochstetter H, Kolb A. Evaporation and condensation of SPH-based fluids[C] //Proceedings of the ACM SIGGRAPH/Eurographics Symposium on Computer Animation. New York: ACM Press, 2017: Article No.3

[14] Clavet S, Beaudoin P, Poulin P. Particle-based viscoelastic fluid simulation[C] //Proceedings of the ACM SIGGRAPH/Eurographics Symposium on Computer Animation. New York: ACM Press, 2005: 219-228

[15] Akinci N, Akinci G, Teschner M. Versatile surface tension and adhesion for SPH fluids[J]. ACM Transactions on Graphics, 2013, 32(6): Article No.182

[16] Xu Shibiao, Zhang Xiaopeng, Chen Yanyun, et al. Interactive simulation for water drop effects[J]. Journal of Com-
puter-Aided Design \& Computer Graphics, 2013, 25(8): 1159-1168(in Chinese)

(徐士彪，张晓鹏，陈彦云，等. 交互式水滴效果模拟[J]. 计 算机辅助设计与图形学学报, 2013, 25(8): 1159-1168)

[17] Hong J M, Kim C H. Discontinuous fluids[J]. ACM Transactions on Graphics, 2005, 24(3): 915-920

[18] Losasso F, Shinar T, Selle A, et al. Multiple interacting liquids[C] //Proceedings of ACM SIGGRAPH 2006 Papers. New York: ACM Press, 2006: 812-819

[19] Thürey N, Wojtan C, Gross M, et al. A multiscale approach to mesh-based surface tension flows[C] //Proceedings of ACM SIGGRAPH 2010 Papers. New York: ACM Press, 2010: Article No.48

[20] Losasso F, Gibou F, Fedkiw R. Simulating water and smoke with an octree data structure[C] //Proceedings of ACM SIGGRAPH 2004 Papers. New York: ACM Press, 2004: 457-462

[21] Setaluri R, Aanjaneya M, Bauer S, et al. SPGrid: a sparse paged grid structure applied to adaptive smoke simulation[J]. ACM Transactions on Graphics, 2014, 33(6): Article No.205

[22] Aanjaneya M, Gao M, Liu H X, et al. Power diagrams and sparse paged grids for high resolution adaptive liquids[J]. ACM Transactions on Graphics, 2017, 36(4): Article No.140

[23] Ferstl F, Westermann R, Dick C. Large-scale liquid simulation on adaptive hexahedral grids[J]. IEEE Transactions on Visualization and Computer Graphics, 2014, 20(10): 1405-1417

[24] Goldade R, Wang Y P, Aanjaneya M, et al. An adaptive variational finite difference framework for efficient symmetric octree viscosity[J]. ACM Transactions on Graphics, 2019, 38(4): Article No.94

[25] Lyu L, Ren X H, Cao W, et al. Adaptive narrow band MultiFLIP for efficient two-phase liquid simulation[J]. Science China Information Sciences, 2018, 61(11): Article No.114101

[26] Enright D, Losasso F, Fedkiw R. A fast and accurate semi-Lagrangian particle level set method[J]. Computers \& Structures, 2005, 83(6/7): 479-490

[27] Harlow F H. The particle-in-cell method for numerical solution of problems in fluid dynamics[R]. United States: Los Alamos Scientific Lab., N. Mex., 1962

[28] Cleary P W, Monaghan J J. Conduction modelling using smoothed particle hydrodynamics[J]. Journal of Computational Physics, 1999, 148(1): 227-264

[29] Lorensen W E, Cline H E. Marching cubes: a high resolution 3D surface construction algorithm[C] //Proceedings of the 14th Annual Conference on Computer Graphics and Interactive Techniques. New York: ACM Press, 1987: 163-169

[30] Chen K C, Chen P S, Wong S K. A heuristic approach to the simulation of water drops and flows on glass panes[J]. Computers \& Graphics, 2013, 37(8): 963-973 